\title{
JACOBI'S BOUND FOR THE ORDER OF SYSTEMS OF FIRST ORDER DIFFERENTIAL EQUATIONS $\left(^{1}\right.$ )
}

\author{
BY \\ BARBARA A. LANDO $\left({ }^{2}\right)$
}

\begin{abstract}
Let $A_{1}, \ldots, A_{n}$ be a system of differential polynomials in the differential indeterminates $y^{(1)}, \ldots, y^{(n)}$, and let $\mathscr{H}$ be an irreducible component of the differential variety $\mathscr{A}\left(A_{1}, \ldots, A_{n}\right)$. If $\operatorname{dim} \mathscr{M}=0$, there arises the question of securing an upper bound for the order of $\mathscr{A}$ in terms of the orders $r_{i j}$ of the polynomials $A_{i}$ in $y^{(j)}$. It has been conjectured that the Jacobi number

$$
J=J\left(r_{i j}\right)=\max \left\{\sum_{i=1}^{n} r_{i j_{i}}: j_{1}, \ldots, j_{n} \text { is a permutation of } 1, \ldots, n\right\}
$$

provides such a bound. In this paper $J$ is obtained as a bound for systems consisting of first order polynomials. Differential kernels are employed in securing the bound, with the theory of kernels obtained in a manner analogous to that of difference kernels as given by R. M. Cohn.
\end{abstract}

1. Introduction. Jacobi investigated the possibility of using $J\left(r_{i j}\right)$ as a bound for the number of arbitrary constants in a solution of a differential system $A_{1}, \ldots$, $A_{n}$. (See Ritt, [5].) The notion of arbitrary constant has since been made more precise with the concept of order of a component [6, Chapter II, $\S 35$ and §10]. Jacobi's bound has been verified for linear systems and for the cases $n=1$ and $n=2$ [5], [6, p. 136], and in this paper is secured for first order systems. For an arbitrary system Ritt [6, p. 135] has obtained a weaker bound $R=\sum_{j=1}^{n} \max \left\{r_{i j}: i=1, \ldots, n\right\}$.

It is also shown here that when $J$ is a bound, it is the best bound in the sense that for any set of integers $r_{i j} \geqq 0, i, j=1, \ldots, n$, there exists a system with orders $r_{i j}$ such that one of the components has order equal to $J\left(r_{i j}\right)$. It may be noted that, in the usual definition of order, $r_{i j}$ is taken to be zero if no derivatives of $y^{(j)}$ of order $\geqq 1$ are effectively present in $A_{i}$; hence $r_{i j}=0$ does not indicate whether or not $y^{(j)}$ itself appears in $A_{i}$. If the definition of order is altered by setting the order equal to $-\infty$ when the indeterminate is not effectively present in the polynomial, a new, possibly lower, Jacobi number $J^{\prime}$ is obtained. $J^{\prime}$ might then be conjectured as a bound.

Received by the editors October 17, 1969.

AMS 1967 subject classifications. Primary 1280; Secondary 1440, 1538, 1615.

Key words and phrases. Differential polynomial, Jacobi bound, differential kernel, specialization, dimension, order of an irreducible differential variety.

( ${ }^{1}$ ) This paper is based on a portion of the author's doctoral dissertation written at Rutgers University under the direction of Professor Richard M. Cohn.

$\left({ }^{2}\right)$ This research was supported in part by National Science Foundation Grant No. GP 8548. 
The theory of differential kernels given here differs from that of difference kernels [2] in the number of distinct generic prolongations and the number of principal realizations. In the difference case these numbers are finite, while in the differential case the generic prolongation and principal realization are unique. The propositions given below for differential kernels hold for difference kernels if statements concerning uniqueness are eliminated: a difference kernel $R$ has a generic prolongation $R^{\prime}$; $\operatorname{ord}_{U} R^{\prime}=\operatorname{ord}_{U} R ; R$ has a principal realization $\alpha$ with the transformal transcendence degree, t.t.d., of $\left.K\langle\alpha\rangle\right|_{K}=\operatorname{deg} R$ and t.d. $\left.K\langle\alpha\rangle\right|_{K\langle V\rangle}$ $=\operatorname{ord}_{U} R$; and for a regular realization $\beta$, t.t.d. $\left.K\langle\beta\rangle\right|_{K} \leqq \operatorname{deg} R$.

The main results of this paper have also been obtained for the difference case (paper in preparation).

2. Notation. The fields considered will be of characteristic zero. The definitions of differential rings, ideals, and varieties are assumed [6]. Let $P$ be a differential ring with a derivation $\partial$. For $a \in P, \partial^{r} a$ is denoted by $a_{r}, r \geqq 0$, with $a_{0}=a$. If there is also an indexing, it will be denoted in superscripts. If $K$ is a differential field and $S$ is a set of elements, $K[S]$ is the ring; $K(S)$, the field; $K\{S\}$, the differential ring; and $K\langle S\rangle$, the differential field obtained by adjoining $S$ to $K$.

If $K$ and $L$ are fields, with $K \subseteq L$, t.d. $\left.L\right|_{K}$ will denote the degree of transcendence of $L$ over $K$. If $K$ and $L$ are differential fields with $K \subseteq L$ and $a \in L$, then $a$ is differentially algebraic over $K$ if there is a nonzero differential polynomial over $K$ with the solution $a$. $a$ is differentially transcendental over $K$ if no such polynomial exists. With the notion of differential algebraic dependence, it can be shown that $L$ has a differential transcendence basis over $K$ [3, pp. 151-156]. The differential transcendence degree of $L$ over $K$, d.t.d. $\left.L\right|_{K}$, is the number of elements in such a basis.

Varieties in the algebraic sense will be denoted by $M$, while differential varieties (also called manifolds) will be denoted by $\mathscr{M}$. (See [6, Chapter II].) If $\mathscr{M}$ is an irreducible variety over the differential field $K$ with a generic zero $\alpha=\left(\alpha^{(1)}, \ldots, \alpha^{(n)}\right)$, then $\operatorname{dim} \mathscr{M}=$ d.t.d. $\left.K\langle\alpha\rangle\right|_{K}$, and ord $\mathscr{M}=$ t.d. $\left.K\langle\alpha\rangle\right|_{K} \leqq \infty$.

Let $P$ be a subring of the field $K$, and let $D$ be a derivation of $P$ into $K$. Let $a^{(1)}, \ldots, a^{(n)} ; b^{(1)}, \ldots, b^{(n)}$ be elements of $K$. Let $\Pi$ be the ideal of polynomials $f(y)=f\left(y^{(1)}, \ldots, y^{(n)}\right)$ in $P\left[y^{(1)}, \ldots, y^{(n)}\right]$ such that $f\left(a^{(1)}, \ldots, a^{(n)}\right)=0$, and let $S$ be a set of generators for $\Pi$. Let $f^{D}(y)$ denote the polynomial of $P\left[y^{(1)}, \ldots, y^{(n)}\right]$ obtained from $f(y)$ by replacing the coefficients of $f(y)$ by their derivatives under $D$. It is well known [3, p. 172] that $D$ can be extended to a derivation $D$ of $P\left[a^{(1)}, \ldots, a^{(n)}\right]$ into $K$ such that $D a^{(i)}=b^{(i)}, i=1, \ldots, n$, if and only if

$$
f^{D}(a)+\sum_{i=1}^{n} \frac{\partial f}{\partial y^{(i)}}(a) \cdot b^{(i)}=0 \quad \text { for all } f(y) \in S .
$$

Furthermore, if the extension exists, it is unique.

Note 1. This result implies the following. Let $K$ and $L$ be fields with $K \subseteq L$, and let $D$ be a derivation of $K$ into $L$. If $D$ is extended to a derivation of $K\left(a^{(1)}, \ldots, a^{(n)}\right)$ 
into $L\left(b^{(1)}, \ldots, b^{(n)}\right)$ with $a^{(i)} \in L$ and $D a^{(i)}=b^{(i)}, i=1, \ldots, n$, then

$$
\text { t.d. }\left.K\left(a^{(1)}, \ldots, a^{(n)}\right)\right|_{K} \geqq \text { t.d. }\left.L\left(b^{(1)}, \ldots, b^{(n)}\right)\right|_{L} .
$$

3. Differential kernels. Let $K$ be a differential field with a derivation $\partial$, and let $a_{i}$ denote the $n$-tuple $\left(a_{i}^{(1)}, \ldots, a_{i}^{(n)}\right), i=0,1, \ldots$, and $a_{0}=a$. A differential kernel $R$ over $K$ consists of an overfield $K\left(a, \ldots, a_{r}\right)$ of $K$ and an extension $D$ of $\partial$ to a derivation of $K\left(a, \ldots, a_{r-1}\right)$ into $K\left(a, \ldots, a_{r}\right)$ such that $D a_{i}=a_{i+1}$, i.e. $D a_{i}^{(j)}=a_{i+1}^{(j)}$, $j=1, \ldots, n . r$ is called the length of the kernel. When $r=0, D$ is taken to be $\partial$.

The degree of transcendence, $\operatorname{deg} R$, of $R$ is defined to be

$$
\text { t.d. }\left.K\left(a, \ldots, a_{r}\right)\right|_{K\left(a, \ldots, a_{:-1}\right)} \text {. }
$$

Let $S$ denote a subindexing of $a$. $S$ is a special set for $R$ if $S_{r}$ is a transcendence basis of $a_{r}$ over $K\left(a, \ldots, a_{r-1}\right)$. Then $\bigcup_{i=0}^{r} S_{i}$ is an algebraically independent set over $K$ (by Note 1 and the derivations of $K\left(a, \ldots, a_{i-1} ; S_{i}\right)$ into $K\left(a, \ldots, a_{i} ; S_{i+1}\right)$ for $i=0, \ldots, r-1)$.

A kernel $R^{\prime}$ is a prolongation of kernel $R$ if $R^{\prime}$ consists of an overfield $K\left(a, \ldots, a_{r+1}\right)$ of $K\left(a, \ldots, a_{r}\right)$ and an extension $D^{\prime}$ of $D$ to a derivation of $K\left(a, \ldots, a_{r}\right)$ into $K\left(a, \ldots, a_{r+1}\right)$ such that $D^{\prime} a_{r}=a_{r+1}$. It follows from Note 1 that if $S$ is a special set for $R$, then $S$ contains a special set for $R^{\prime}$, and that t.d. $\left.K\left(a, \ldots, a_{r}\right)\right|_{K\left(a, \ldots, a_{r-i}\right)}$ $\geqq$ t.d. $\left.K\left(a, \ldots, a_{r+1}\right)\right|_{K\left(a, \ldots, a_{r}\right)}$. Thus $\operatorname{deg} R \geqq \operatorname{deg} R^{\prime} . R^{\prime}$ is called a generic prolongation if $\operatorname{deg} R=\operatorname{deg} R^{\prime}$. In this case a special set for $R$ is a special set for $R^{\prime}$.

A kernel $\bar{R}$ consisting of $K\left(\bar{a}, \ldots, \bar{a}_{r}\right)$ and $\bar{D}$ is a specialization of $R$ if $\left(\bar{a}, \ldots, \bar{a}_{r}\right)$ is a specialization of $\left(a, \ldots, a_{r}\right)$ over $K$ in the algebraic sense. We note that if $\phi$ is the homomorphism of $K\left[a, \ldots, a_{r}\right]$ onto $K\left[\bar{a}, \ldots, \bar{a}_{r}\right]$, then

$$
\phi\left(D a_{i}^{(j)}\right)=\phi a_{i+1}^{(j)}=\bar{a}_{i+1}^{(j)}=\bar{D}\left(\bar{a}_{i}^{(j)}\right)=\bar{D}\left(\phi a_{i}^{(j)}\right),
$$

and $\phi$ is necessarily a specialization in the differential sense.

Proposition 1. Let $R$ be a kernel. There is a generic prolongation $R^{\prime}$ of $R$. Furthermore, if $\bar{R}$ is any other prolongation of $R, \bar{R}$ is a specialization of $R^{\prime}$.

Proof. Let $R$ consist of $K\left(a, \ldots, a_{r}\right)$ and $D$, and let $s=\operatorname{deg} R$. Let $S$ be a special set. Let $S_{r+1}$ be a set of $s$ elements which are algebraically independent over $K\left(a, \ldots, a_{r}\right)$. There is a derivation $D$ of $K\left(a, \ldots, a_{r-1} ; S_{r}\right)$ into $K\left(a, \ldots, a_{r} ; S_{r+1}\right)$ extending $D$, with $D a_{r}^{(j)}=a_{r+1}^{(j)}$, where $a_{r+1}^{(j)}$ is a distinct element of $S_{r+1}$ for each $j$. Since $K\left(a, \ldots, a_{r}\right)$ is a separable algebraic extension of $K\left(a, \ldots, a_{r-1} ; S_{r}\right), D$ can be extended in a unique way to a derivation $D$ of $K\left(a, \ldots, a_{r}\right)$ into $K\left(a, \ldots, a_{r} ; S_{r+1}\right)$ [3, p. 175]. For $j=1, \ldots, n$, let $a_{r+1}^{(j)}=D a_{r}^{(j)}$. Then $K\left(a, \ldots, a_{r+1}\right)$ with $D$ is a kernel $R^{\prime}$, and $\operatorname{deg} R^{\prime}=\operatorname{deg} R$.

Let $K\left(a, \ldots, a_{r}, \bar{a}_{r+1}\right), \bar{D}$, be a prolongation $\bar{R}$ of $R$. Since $S_{r+1}$ is an algebraically independent set over $K\left(a, \ldots, a_{r}\right)$, there is a homomorphism $\phi$ of $K\left(a, \ldots, a_{r}\right)\left[S_{r+1}\right]$ onto $K\left(a, \ldots, a_{r}\right)\left[\bar{S}_{r+1}\right]$, where $\bar{S}_{r+1}=\bar{D} S_{r}$. We note that if $c \in K\left(a, \ldots, a_{r-1} ; S_{r}\right)$, $D c \in K\left(a, \ldots, a_{r}\right)\left[S_{r+1}\right]$. Now if $a_{r}^{(j)} \notin S_{r}$, it is separably algebraic over

$$
K\left(a, \ldots, a_{r-1} ; S_{r}\right)
$$


with minimal polynomial $f(y)$, and

$$
D a_{r}^{(j)}=a_{r+1}^{(j)}=-\left(f^{D}\left(a_{r}^{(j)}\right) / f^{\prime}\left(a_{r}^{(j)}\right)\right) \in K\left(a, \ldots, a_{r}\right)\left[S_{r+1}\right] .
$$

By a similar argument,

$$
\bar{D} a_{r}^{(j)}=\bar{a}_{r+1}^{(j)}=-\left(f^{\bar{D}}\left(a_{r}^{(j)}\right) / f^{\prime}\left(a_{r}^{(j)}\right)\right) \in K\left(a, \ldots, a_{r}\right)\left[\bar{S}_{r+1}\right] .
$$

Hence $\phi$ is already defined on $a_{r+1}^{(j)}$ with $a_{r+1}^{(j)}=\bar{a}_{r+1}^{(j)}$, and thus provides the required specialization.

COROLlARY. The generic prolongation of a differential kernel is unique in the sense of isomorphism.

Let $U$ be a subindexing of $a$ such that $U$ contains a special set and

$$
\text { t.d. }\left.K\left(U, \ldots, U_{r}\right)\right|_{K\left(U, \ldots, U_{r-1}\right)}=\operatorname{deg} R .
$$

Then the order of $R$ with respect to $U$, ord $d_{U}$, is defined to be

$$
\text { t.d. }\left.K\left(a, \ldots, a_{r}\right)\right|_{K\left(U, \ldots, U_{r}\right)}
$$

If $U$ is itself a special set, then ord $R$ is defined, and

$$
\begin{aligned}
\operatorname{ord}_{U} R & =\text { t.d. }\left.K\left(a, \ldots, a_{r}\right)\right|_{K\left(U, \ldots, U_{r}\right)}=\text { t.d. }\left.K\left(a, \ldots, a_{r-1} ; U_{r}\right)\right|_{K\left(U, \ldots, U_{r}\right)} \\
& =\text { t.d. }\left.K\left(a, \ldots, a_{r-1}\right)\right|_{K\left(U, \ldots, U_{r-1}\right)}
\end{aligned}
$$

If $\operatorname{deg} R=0, U$ may be the empty set, and we define the order of $R$, ord $R$, to be t.d. $\left.K\left(a, \ldots, a_{r}\right)\right|_{K}$.

Proposition 2. Let $R$ be a kernel with a generic prolongation $R^{\prime}$. Let $U$ be a subindexing of a such that $\operatorname{ord}_{U} R$ is defined. Then $\operatorname{ord}_{U} R^{\prime}$ is defined, and

$$
\operatorname{ord}_{U} R=\operatorname{ord}_{U} R^{\prime} \text {. }
$$

Proof. Analogous to that of Lemma III, Chapter 6, of [2].

If $R: K\left(a, \ldots, a_{r}\right), D$, is a kernel, and $\alpha=\left(\alpha^{(1)}, \ldots, \alpha^{(n)}\right)$ is contained in a differential overfield of $K$, then $\alpha$ is a realization of $R$ if $\left(\alpha, \alpha_{1}, \ldots, \alpha_{r}\right)$ is a specialization of $\left(a, a_{1}, \ldots, a_{r}\right)$ over $K$. If the specialization is generic, the realization is called regular. Finally, $\alpha$ is called a principal realization of $R$ if there is a sequence of kernels $R=R^{(0)}, R^{(1)}, \ldots$, each a generic prolongation of the preceding, such that $\alpha$ is a regular realization of each $R^{(k)}, k=0,1, \ldots$

Proposition 3. Every kernel $R$ has a principal realization $\alpha$ which is unique in the sense of isomorphism. d.t.d. $\left.K\langle\alpha\rangle\right|_{K}=\operatorname{deg} R$; and if $U$ is a subindexing of a such that $\operatorname{ord}_{U} R$ is defined and $V$ is the corresponding subindexing of $\alpha$, then t.d. $\left.K\langle\alpha\rangle\right|_{K\langle V\rangle}$ $=\operatorname{ord}_{U} R$. If $U$ is a special set, $V$ is a differential transcendence basis for $\alpha$ over $K$.

Proof. Proof of existence is analogous to that of Lemma V, Chapter 6, of [2]. The uniqueness of $\alpha$ follows from the uniqueness of generic prolongations in the differential case. 
Proposition 4. If $\alpha$ is a regular realization of $R$, then d.t.d. $\left.K\langle\alpha\rangle\right|_{K} \leqq \operatorname{deg} R$ with equality holding if and only if $\alpha$ is a principal realization.

Proof. Analogous to that of Lemma VI, Chapter 6, of [2].

COROLlary. A realization $\beta$ of a kernel $R$ which specializes to a principal realization $\alpha$ is a principal realization, and the specialization is generic.

Proof. Clearly $\beta$ is a regular realization, and d.t.d. $\left.K\langle\beta\rangle\right|_{K} \geqq$ d.t.d. $\left.K\langle\alpha\rangle\right|_{K}=$ $\operatorname{deg} \boldsymbol{R}$. By the proposition, $\beta$ is a principal realization. By the uniqueness of principal realizations, $K\langle\beta\rangle \cong K\langle\alpha\rangle$.

In general, however, if $\alpha$ is any realization of $R$, it is not necessarily true that d.t.d. $\left.K\langle\alpha\rangle\right|_{K} \leqq \operatorname{deg} R$.

EXAMPLE 1. Let $a=(u, v, w)$ where $u, v$, and $w$ are algebraically independent over $K$. Let $a_{1}=\left(u, v, w_{1}\right)$ where $w_{1}$ is a zero of the polynomial $v y-u$. Then $K\left(a, a_{1}\right)$ defines a kernel $R$ of degree 0 . Let $a^{\prime}=(0,0, w)$ and $a_{1}^{\prime}=\left(0,0, w_{1}^{\prime}\right)$ where $w_{1}^{\prime}$ is transcendental over $K(w) . K\left(a^{\prime}, a_{1}^{\prime}\right)$ defines a kernel $R^{\prime}$ of degree $1 . R$ specializes to $R^{\prime}$, but $\operatorname{deg} R<\operatorname{deg} R^{\prime}$. If $\alpha^{\prime}$ is a principal realization of $R^{\prime}$, it is a realization, but not a regular one, of $R$. Thus $\operatorname{deg} R=0$, but $R$ has a realization $\alpha^{\prime}$ with

$$
\text { d.t.d. }\left.K\left\langle\alpha^{\prime}\right\rangle\right|_{K}=1 \text {. }
$$

Note 2. If $R$ is a kernel of length $r>0, R$ is equivalent to a kernel $R^{\prime}$ of length 1 in the sense that their realizations generate the same differential field extensions of $K$. If $R$ consists of $K\left(a, \ldots, a_{r}\right)$ and $D$, with $r>1$, let $b$ be the vector $\left(a, \ldots, a_{r-1}\right)$ having $r n$ components and let $b_{1}=\left(a_{1}, \ldots, a_{r}\right)$. Then $D b=b_{1}$, and $R^{\prime}$ consisting of $K\left(b, b_{1}\right)$ and $D$ is a kernel of length 1 which is equivalent to $R$.

4. Specialization problem. Let $R$ and $\bar{R}$ be kernels with fields $K\left(a, \ldots, a_{r}\right)$ and $K\left(\bar{a}, \ldots, \bar{a}_{r}\right)$, respectively, $r \geqq 0$, such that $K\left(a, \ldots, a_{r-1}\right) \cong_{K} K\left(\bar{a}, \ldots, \bar{a}_{r-1}\right)$. (When $r=0$, the isomorphism is the identity automorphism of $K$.) Let $\bar{\alpha}$ be a principal realization of $\bar{R}$. It will be shown that if $\bar{R}$ is a specialization of $R$ over $K$, then $\bar{\alpha}$ is the specialization of a principal realization $\alpha$ of $R$.

In general, a specialization of kernels does not imply a specialization of principal realizations. In Example $1 R$ specializes to $R^{\prime}$, but $\operatorname{deg} R<\operatorname{deg} R^{\prime}$, and hence there is no specialization of principal realizations. Furthermore, while $\operatorname{deg} R \geqq \operatorname{deg} R^{\prime}$ is a necessary condition for the existence of a specialization of principal realizations, it is not sufficient.

EXAMPLE 2. Let $R$ consist of $K\left(a, a_{1}\right), D$, where $a$ is a single element algebraically independent over $K$, and $a_{1}$ is a zero of $f(y)=y^{2}-4 a$. Let $R^{\prime}$ consist of $K(0,0)$ and $D^{\prime}$. Then $R$ specializes to $R^{\prime}$, and $\operatorname{deg} R=\operatorname{deg} R^{\prime}=0 . \quad D a_{1}=-f^{D}\left(a_{1}\right) / f^{\prime}\left(a_{1}\right)$ $=4 a_{1} / 2 a_{1}=2$. Since $\left(a, a_{1}, 2\right)$ does not specialize to $(0,0,0)$, the principal realization of $R$ does not specialize to the principal realization (0) of $R^{\prime}$.

The lemmas below are concerned with arbitrary fields, not necessarily differential fields. 
LeMma 1. Let $K$ and $L$ be fields; let $K[a]=K\left[a^{(1)}, \ldots, a^{(n)}\right]$ be an integral domain and $M$ a field containing $K$. Let $\sigma: K[a] \rightarrow L$ and $\tau: M \rightarrow L$ be homomorphisms over $K$ with restrictions $\left.\sigma\right|_{K}=\left.\tau\right|_{K} \neq 0$. Then there exists a free join $M^{\prime}\left[a^{\prime}\right]$ of $M$ and $K[a]$ over $K$ with $\psi_{1}: K\left[a^{\prime}\right] \cong_{K} K[a]$ and $\psi_{2}: M^{\prime} \cong_{K} M$, and there exists a homomorphism $\phi: M^{\prime}\left[a^{\prime}\right] \rightarrow L$ such that $\left.\phi\right|_{K\left[a^{\prime}\right]}=\sigma \circ \psi_{1}$ and $\left.\phi\right|_{M^{\prime}}=\tau \circ \psi_{2}$.

Proof. Since $K$ is a field and $\sigma \neq 0$, we may assume without loss of generality that $K \subseteq L$ and that $\left.\sigma\right|_{K}=\left.\tau\right|_{K}=\mathrm{id}_{K}$. By the universal mapping property of tensor products, there exists a homomorphism $\Phi$ of $M \otimes_{K} K[a]$ into $L$ such that $\left.\Phi\right|_{K[a]}=\sigma$ and $\left.\Phi\right|_{M}=\tau$.

Let $\Pi$ be the kernel of $\Phi$. Since $0 \in \Pi$ and $\Pi$ is prime, there exists a prime divisor $\Lambda$ of the ideal (0) such that $\Lambda \subseteq \Pi . M \otimes K[a]$ is noetherian; thus, by Corollary 3 to Theorem 10, Chapter IV, of [7], $\Lambda$ consists of zero divisors. Hence $M \otimes K[a] / \Lambda$ is a free join of $M$ and $K[a]$ over $K\left[7\right.$, p. 189], and $M \otimes K[a] / \Lambda \cong{ }_{K} M^{\prime}\left[a^{\prime}\right]$ with $\psi_{1}: K\left[a^{\prime}\right] \cong_{K} K[a]$ and $\psi_{2}: M^{\prime} \cong_{K} M$. Since $\Lambda \subseteq \Pi$, there is a homomorphism $\phi: M^{\prime}\left[a^{\prime}\right] \rightarrow L$ determined by $\Phi$ such that $\left.\phi\right|_{K\left[a^{\prime}\right]}=\sigma \circ \psi_{1}$ and $\left.\phi\right|_{M^{\prime}}=\tau \circ \psi_{2}$.

It may be assumed in the above lemma that either $\psi_{1}=\mathrm{id}$ or $\psi_{2}=\mathrm{id}$.

LEMMA 2. Let $L$ be an algebraically closed field and $L(b)=L\left(b^{(1)}, \ldots, b^{(n)}\right)$ with t.d. $\left.L(b)\right|_{L}=1$. Let $\bar{b}=\left(\bar{b}^{(1)}, \ldots, \bar{b}^{(n)}\right)$ consist of elements of $L$. If $b$ specializes to $\bar{b}$ over $L$, then there exists a parameter $t \in L(b)$, transcendental over $L$, such that $L[b]$ has a representation in the power series ring $L[[t]]$ with

$$
b^{(i)}=\bar{b}^{(i)}+\sum_{j=1}^{\infty} c_{i j} t^{j}, \quad i=1, \ldots, n .
$$

Proof. $L(b)$ is an algebraic function field of one variable over $L$. Using the notation and results of Chevalley [1], we may obtain a place $\mathfrak{p}$ and a valuation ring $\mathfrak{o}$ of $L(b)$ over $L$ with $L[b] \subseteq \mathfrak{o}$ and $b^{(i)}-\bar{b}^{(i)} \in \mathfrak{p}, i=1, \ldots, n,[1$, Chapter I, Corollary 1 to Theorem 1]. By Theorem 2, Chapter I, of [1], $\mathfrak{o} / \mathfrak{p}$ is algebraic over $L$; hence $\mathfrak{o} / \mathfrak{p}=L$ since $L$ is algebraically closed.

Since $\mathfrak{o} / \mathfrak{p}=L$ is separable over $L$, it follows that every element $c$ in the $\mathfrak{p}$-adic completion of $L(b)$ has a representation $c=\sum_{j=r}^{\infty} c_{j} t^{j}$ with $r$ an integer and $c_{j} \in L$ for all $j$ [1, p. 46]. Moreover, if $c \in \mathfrak{o}$, then $r \geqq 0$; and if $c \in \mathfrak{p}$, then $r \geqq 1$. Then, since $L[b] \subseteq \mathfrak{0}, L[b] \subseteq L[[t]]$, and since $b^{(i)}-\bar{b}^{(i)} \in \mathfrak{p}, b^{(i)}-\bar{b}^{(i)}=\sum_{j=1}^{\infty} c_{i j} t^{j}$ with $\bar{b}^{(i)}, c_{i j} \in L$. This completes the proof.

Let $K\left[\left[t, t_{1}, \ldots, t_{m}\right]\right]$ be the formal power series ring in $m+1$ indeterminates $t_{0}=t, t_{1}, \ldots, t_{m}$. For $p \in K\left[\left[t, \ldots, t_{m}\right]\right], p$ may be written $p_{0}+p_{1}+\cdots+p_{k}+\cdots$, where $p_{k}=0$ or is a homogeneous polynomial of degree $k$ in $K\left[t, \ldots, t_{m}\right]$.

Lemma 3. Let $D$ be a derivation of $K$ into $K[[t]]$. Then $D$ can be extended to a unique derivation $D$ of $K\left[\left[t, \ldots, t_{m-1}\right]\right]$ into $K\left[\left[t, \ldots, t_{m}\right]\right]$ such that $D t_{j}=t_{j+1}$ and for $p=p_{0}+p_{1}+\cdots+p_{k}+\cdots$ in $K\left[\left[t, \ldots, t_{m-1}\right]\right], D p=\sum_{k=0}^{\infty} D p_{k}$.

Proof. $D$ can be uniquely extended to a derivation $D$ of $K\left[t, \ldots, t_{m-1}\right]$ into 
$K\left[\left[t, \ldots, t_{m}\right]\right]$ with $D t_{j}=t_{j+1}, j=0, \ldots, m-1$. If $\mu=a t^{k_{0}} \cdots t_{m-1}^{k_{m}-1}$ is a monomial of degree $k=\sum_{j=0}^{m-1} k_{j}$,

$$
D \mu=(D a) \cdot \prod_{j=0}^{m-1} t_{j}^{k_{j}}+a \cdot \sum_{j=0}^{m-1}\left(k_{j} t_{j}^{k_{j}-1} \cdot t_{j+1} \cdot \prod_{i \neq j} t_{i}^{k_{i}}\right),
$$

where $D a \in K[[t]]$. Thus $D \mu$ is a power series of $K\left[\left[t, \ldots, t_{m}\right]\right]$ of order $\geqq k$. If $p=p_{0}+p_{1}+\cdots \in K\left[\left[t, \ldots, t_{m-1}\right]\right]$, then ord $D p_{k} \geqq k$, and hence the sum $\sum_{k=0}^{\infty} D p_{k}$ converges to a series in $K\left[\left[t, \ldots, t_{m}\right]\right]$. Thus we may define $D p$ to be the series $\sum_{k=0}^{\infty} D p_{k}$. It is easily shown that $D$ is a derivation on $K\left[\left[t, \ldots, t_{m-1}\right]\right]$ and it is clear that $D$ is unique.

Now let $K$ be a differential field with derivation $\partial$. Let $a$ represent $\left(a^{(1)}, \ldots, a^{(n)}\right)$.

THEOREM 1. Let $R$ and $\bar{R}$ be differential kernels consisting of $K\left(a, \ldots, a_{r}\right), D$, and $K\left(\bar{a}, \ldots, \bar{a}_{r}\right), \bar{D}$, respectively, $r \geqq 0$, with $K\left(a, \ldots, a_{r-1}\right) \cong{ }_{K} K\left(\bar{a}, \ldots, \bar{a}_{r-1}\right)$. Let $\alpha$ and $\bar{\alpha}$ be principal realizations of $R$ and $\bar{R}$, respectively. If $\bar{R}$ is a specialization of $R$, then $\bar{\alpha}$ is a differential specialization of $\alpha$.

Proof. Since principal realizations are unique up to isomorphism, it suffices to prove the result for any principal realizations of $R$ and $\bar{R}$. Also, we may assume that the kernels are of length 0 or 1 (Note 2). The proof is given first for the case of length 1 .

$R$ and $\bar{R}$ have fields $K\left(a, a_{1}\right)$ and $K\left(\bar{a}, \bar{a}_{1}\right)$, respectively. Since $K(a) \cong K(\bar{a})$, we may assume that $a=\bar{a}$. Since $\left(a_{1}\right)$ specializes to $\left(\bar{a}_{1}\right)$ over $K(a), \operatorname{deg} R \geqq \operatorname{deg} \bar{R}$. If $\operatorname{deg} R$ $=\operatorname{deg} \bar{R}$, then $K\left(a, a_{1}\right) \cong K\left(a, \bar{a}_{1}\right)$, and by the uniqueness of principal realizations, $K\{\alpha\} \cong K\{\bar{\alpha}\}$. It remains to consider the case $\operatorname{deg} R>\operatorname{deg} \bar{R}$.

It will be shown that we may assume that $\operatorname{deg} R=\operatorname{deg} \bar{R}+1$. Let

$$
t=\text { t.d. }\left.K\left(a, a_{1}\right)\right|_{K(a)} \quad \text { and } \quad s=\text { t.d. }\left.K\left(a, \bar{a}_{1}\right)\right|_{K(a)}, \quad t>s .
$$

There exists a sequence of specializations over $K(a)$ :

$$
a_{1}=b_{(t)} \rightarrow b_{(t-1)} \rightarrow \cdots \rightarrow b_{(s)}=\bar{a}_{1}
$$

with t.d. $\left.K\left(a, b_{(i)}\right)\right|_{K(a)}=i, i=s, \ldots, t[8$, p. 194]. Since there is a derivation $D: K(a) \rightarrow K\left(a, a_{1}\right)$ and since $a_{1} \overrightarrow{K(a)} b_{(i)}$, there is a derivation $D^{(i)}: K(a) \rightarrow K\left(a, b_{(i)}\right)$, $i=s, \ldots, t$. Thus each $K\left(a, b_{(i)}\right), D^{(i)}$, is a kernel of degree $i$, and it suffices to prove the theorem for the case $\operatorname{deg} R=\operatorname{deg} \bar{R}+1$.

Next it is shown that we may assume that $\operatorname{deg} \bar{R}=0$. Suppose $\operatorname{deg} \bar{R}=s>0$ and $\operatorname{deg} R=s+1$. Let $K\langle\bar{\alpha}\rangle, \bar{\partial}$, and $K\langle\alpha\rangle, \partial$, be the differential fields generated by the principal realizations $\bar{\alpha}$ and $\alpha$. By Proposition 3, d.t.d. $\left.K\langle\bar{\alpha}\rangle\right|_{K}=s$ and d.t.d. $\left.K\langle\alpha\rangle\right|_{K}$ $=s+1$. We may assume $\left(\bar{\alpha}, \bar{\alpha}_{1}\right)=\left(a, \bar{a}_{1}\right)$ and $\left(\alpha, \alpha_{1}\right)=\left(a, a_{1}\right)$. Since $\left(a, a_{1}\right)$ specializes to $\left(a, \bar{a}_{1}\right)$ over $K$, there is a subindexing $S$ of $a$ such that $S$ is a special set for $\bar{R}$ and is contained in a special set for $R$. For $j \geqq 1$, let $\bar{\partial}^{j} S$ be denoted by $\bar{S}_{j}$ and $\partial^{j} S$ by $S_{j}$. Since the realizations are principal, $\bigcup_{i=1}^{\infty} \bar{S}_{i}$ and $\bigcup_{i=1}^{\infty} S_{i}$ are both algebraically independent sets over $K(a)$, and thus $K\left(a ; S_{1}, S_{2}, \ldots\right) \cong K\left(a ; \bar{S}_{1}, \bar{S}_{2}, \ldots\right)$. By 
taking a principal realization isomorphic to $\bar{\alpha}$ we may assume that $S_{i}=\bar{S}_{i}$ for all $i \geqq 0 . K\langle S\rangle$ is a differential field; $K\langle S\rangle\left(a, a_{1}\right)$ is a kernel $R^{\prime}$ over $K\langle S\rangle$ of degree 1 with a principal realization $\alpha$; and $K\langle S\rangle\left(a, \bar{a}_{1}\right)$ is a kernel $\bar{R}^{\prime}$ over $K\langle S\rangle$ of degree 0 with a principal realization $\bar{\alpha}$. The specialization $K\left[a, a_{1}\right] \rightarrow K\left[a, \bar{a}_{1}\right]$ may be extended to the homomorphism $K\left(S, S_{1}\right)\left[a, a_{1}\right] \rightarrow K\left(S, S_{1}\right)\left[a, \bar{a}_{1}\right]$, and then to $K\langle S\rangle\left[a, a_{1}\right] \underset{K\langle S\rangle}{\longrightarrow} K\langle S\rangle\left[a, \bar{a}_{1}\right]$ since $\bigcup_{i=2}^{\infty} S_{i}$ is an algebraically independent set

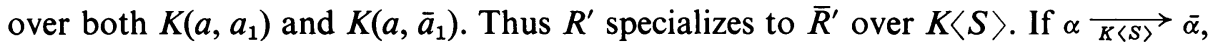
then clearly $\alpha \vec{K} \quad \bar{\alpha}$. Thus it suffices to consider the case $\operatorname{deg} \bar{R}=0$.

Let $L$ be the algebraic closure of $K\left(a, \bar{a}_{1}\right)$ (and hence of $K(a)$ ). Since there exist homomorphisms $K(a)\left[a_{1}\right] \underset{K(a)}{\longrightarrow} K(a)\left[\bar{a}_{1}\right] \subseteq L$ and $L \stackrel{\text { id }}{\rightarrow} L$, by Lemma 1 there exists a free join $L\left[a_{1}^{\prime}\right]$ of $L$ and $K(a)\left[a_{1}\right]$ over $K(a)$ with $K(a)\left[a_{1}^{\prime}\right] \cong K(a)\left[a_{1}\right]$, and there exists a homomorphism $\phi: L\left[a_{1}^{\prime}\right] \vec{L} L$ with $\phi a_{1}^{\prime}=\bar{a}_{1}$. We may assume that $a_{1}^{\prime}=a_{1}$ (by taking a kernel isomorphic to $R$ over $K(a))$. Thus $\phi$ gives a specialization of $a_{1}$ onto $\bar{a}_{1}$ over $L$.t.d. $\left.L\left(a_{1}\right)\right|_{L}=1$ since t.d. $\left.K\left(a, a_{1}\right)\right|_{K(a)}=1$, and $L\left(a_{1}\right)$ is a free join over $K(a)$. By Lemma 2 there exists $t \in L\left(a_{1}\right)$, transcendental over $L$, such that $L\left[a_{1}\right]$ has a representation in $L[[t]]$ with

$$
a_{1}^{(i)}=\bar{a}_{1}^{(i)}+\sum_{j=1}^{\infty} c_{i j} t^{j} \text { for } i=1, \ldots, n .
$$

Since $L$ is algebraic over $K(a)$, the derivations $D: K(a) \rightarrow K(a)\left[a_{1}\right] \subseteq L[[t]]$ and $\bar{D}: K(a) \rightarrow K(a)\left[\bar{a}_{1}\right] \subseteq L$ have unique extensions to $D: L \rightarrow L[[t]]$ and $\bar{D}: L \rightarrow L$ [3, pp. 172-175]. By Lemma 3, $D$ can be extended successively to derivations

$$
\begin{aligned}
D_{1}: L[[t]] & \rightarrow L\left[\left[t, t_{1}\right]\right], \\
D_{2}: L\left[\left[t, t_{1}\right]\right] & \rightarrow L\left[\left[t, t_{1}, t_{2}\right]\right], \ldots, \\
D_{j}: L\left[\left[t, \ldots, t_{j-1}\right]\right] & \rightarrow L\left[\left[t, \ldots, t_{j}\right]\right], \ldots .
\end{aligned}
$$

For each $j, t_{j}$ is transcendental over $L\left[\left[t, \ldots, t_{j-1}\right]\right] ; D_{j} t_{j-1}=t_{j}$; and if $q=q_{0}+q_{1}$ $+\cdots+q_{k}+\cdots \in L\left[\left[t, \ldots, t_{j-1}\right]\right]$ with $q_{k}$ homogeneous of degree $k$, then $D_{j} q=$ $\sum_{k=0}^{\infty} D_{j} q_{k}$. Then $\bigcup_{j=0}^{\infty} L\left[\left[t, \ldots, t_{j}\right]\right]$ is a differential ring $P$ with a derivation $D$ obtained by taking the union of the derivations $D_{j}, j=0,1, \ldots$

$a_{1}=D a \in L[[t]]$. For $h>1$, let $a_{h}=D^{h} a$. Then $K\left[a, a_{1}, \ldots\right]$ is a differential subring of $P$, and $K\left(a, a_{1}, \ldots\right)$ is a differential field. $\left(a, a_{1}, \ldots, a_{h}, \ldots\right)$ is a regular realization of $R$, and d.t.d. $\left.K\left(a, a_{1}, \ldots\right)\right|_{K} \leqq \operatorname{deg} R=1$. Since $t \in L\left(a_{1}\right), t_{h-1}=$ $D^{h-1} t \in L\left(a_{1}, \ldots, a_{h}\right)$ for any $h>0 . t_{h-1}$ is transcendental over $L\left[\left[t, \ldots, t_{h-2}\right]\right]$ and therefore over $L\left[a, \ldots, a_{h-1}\right]$. Hence for all $h>0$,

$$
\text { t.d. }\left.K\left(a, \ldots, a_{h}\right)\right|_{K\left(a, \ldots, a_{h-1}\right)} \geqq \text { t.d. }\left.L\left(a_{1}, \ldots, a_{h}\right)\right|_{L\left(a_{1}, \ldots, a_{h-1}\right)} \geqq 1 \text {, }
$$

and thus d.t.d. $\left.K\left(a, a_{1}, \ldots\right)\right|_{K} \geqq 1$. Therefore d.t.d. $\left.K\left(a, a_{1}, \ldots\right)\right|_{K}=1=\operatorname{deg} R$, and $\left(a, a_{1}, a_{2}, \ldots\right)$ is a principal realization of $R$ (by Proposition 4).

$L$ is a differential field with the derivation $\bar{D} . \bar{D} a=\bar{a}_{1}$. For $h>1$ let $\bar{a}_{h}=\bar{D}^{h} a$. Then $\left(a, \bar{a}_{1}, \bar{a}_{2}, \ldots\right)$ is a regular realization of $\bar{R}$. Since $0 \leqq$ d.t.d. $\left.K\left(a, \bar{a}_{1}, \ldots\right)\right|_{K}$ $\leqq \operatorname{deg} \bar{R}=0,\left(a, \bar{a}_{1}, \bar{a}_{2}, \ldots\right)$ is a principal realization of $\bar{R}$. 
Let $\Phi: P \rightarrow L$ be defined by $\Phi t_{j}=0, j=0,1, \ldots$ Then $\Phi$ is a homomorphism over $L$ and from (1) it is clear that $\Phi a_{1}=\bar{a}_{1}=\phi a_{1}$. Thus $\Phi$ is an extension of the specialization $\phi: L\left[a_{1}\right] \rightarrow L\left[\bar{a}_{1}\right]=L$ (and of the specialization $K\left[a, a_{1}\right] \rightarrow K\left[a, \bar{a}_{1}\right]$ ), and for $c \in K[a], \Phi(D c)=\phi(D c)=\bar{D} c$. We will show that $\Phi \circ D=\bar{D} \circ \Phi$ on $P$. First for $p \in L$, let $f(y)$ be the minimal polynomial of $p$ over $K(a)$. Then $D p=$ $-f^{D}(p) / f^{\prime}(p)$, and $\bar{D} p=-f^{D}(p) / f^{\prime}(p)$. We may assume that $f(y)$ has coefficients in $K[a]$; then $\Phi\left(f^{D}(p)\right)=f^{D}(\Phi p)$. Using this and the fact that $\Phi p=p$, we obtain

$$
\begin{aligned}
\Phi(D p) & =\Phi\left(-f^{D}(p) / f^{\prime}(p)\right)=-f^{\bar{D}}(\Phi p) / f^{\prime}(\Phi p) \\
& =-f^{\bar{D}}(p) / f^{\prime}(p)=\bar{D} p=\bar{D}(\Phi p) .
\end{aligned}
$$

For $q \in L\left[\left[t, \ldots, t_{h}\right]\right], q=q_{0}+q^{\prime}$, where $q_{0} \in L$, and $q^{\prime}=0$ or each term of $q^{\prime}$ involves $t$ 's. $D q=D q_{0}+D q^{\prime}$, where $D q^{\prime}=0$ or each term of $D q^{\prime}$ involves some $t$ 's from among $t, t_{1}, \ldots, t_{h+1}$. Then

$$
\Phi(D q)=\Phi\left(D q_{0}+D q^{\prime}\right)=\Phi\left(D q_{0}\right)=\bar{D}\left(q_{0}\right)=\bar{D}(\Phi q)
$$

for any $q \in P$.

The restriction of $\Phi$ to $K\left[a, a_{1}, \ldots\right]$ is a homomorphism into $L$ with $\Phi a=a$ and $\Phi a_{1}=\bar{a}_{1}$. Assume that it has been shown that $\Phi a_{j}=\bar{a}_{j}, j=0, \ldots, h-1$. Then using (2) we obtain $\Phi a_{h}=\Phi\left(D a_{n-1}\right)=\bar{D}\left(\Phi a_{n-1}\right)=\bar{D} \bar{a}_{n-1}=\bar{a}_{h}$. Therefore, by induction, $\Phi$ provides a specialization of the principal realization $\left(a, a_{1}, \ldots, a_{h}, \ldots\right)$ of $R$ to the principal realization $\left(a, \bar{a}_{1}, \ldots\right)$ of $\bar{R}$.

The preceding proof can be modified easily for the case of kernels of length 0 . The same assumptions can be made: that $\operatorname{deg} R=1$ and $\operatorname{deg} \bar{R}=0$, and that $a$ specializes to $\bar{a}$ over the algebraic closure $M$ of $K(\bar{a})$. Then $M[a]$ has a representation in $M[[t]]$ with $a^{(i)}=\bar{a}^{(i)}+\sum_{j=1}^{\infty} d_{i j} t^{j}$; and $\bigcup_{h=1}^{\infty} M\left[\left[t, \ldots, t_{h}\right]\right], D$, and $\Phi$ can be defined in a similar manner. However, in this case, since $M$ is the algebraic closure of $K, D=\bar{D}$ on $M$. Thus for $h=1,2, \ldots$,

$$
D a_{h-1}^{(i)}=\bar{a}_{h}^{(i)}+D^{h}\left(\sum_{j=1}^{\infty} d_{i j} t^{j}\right)
$$

and it is clear that $\Phi a_{h}=\bar{a}_{h}, h \geqq 0$.

COROLLARY. Every regular realization of a kernel $R$ is the specialization of $a$ principal realization.

Proof. Let $R$ be a kernel of length $r \geqq 0$, and let $\beta$ be a regular realization of $R$. By Proposition 4, d.t.d. $\left.K\langle\beta\rangle\right|_{K} \leqq \operatorname{deg} R$. We use induction on

$$
m=\operatorname{deg} R \text {-d.t.d. }\left.K\langle\beta\rangle\right|_{K} \text {. }
$$

Basis. $m=0$. Then d.t.d. $\left.K\langle\beta\rangle\right|_{K}=\operatorname{deg} R$, and $\beta$ is a principal realization.

Inductive step. Assume the result holds for any kernel $\bar{R}$ with a regular realization $\bar{\beta}$ such that $0 \leqq \operatorname{deg} \bar{R}-$ d.t.d. $\left.K\langle\bar{\beta}\rangle\right|_{K}<M$.

Let $\operatorname{deg} R$-d.t.d. $\left.K\langle\beta\rangle\right|_{K}=M>0$. Let $R_{k}$ be the kernel $K\left(\beta, \ldots, \beta_{r+k}\right), k \geqq 0$. Then $\operatorname{deg} R \geqq \operatorname{deg} R_{1} \geqq \cdots \geqq \operatorname{deg} R_{k} \geqq 0$. Let $k$ be the minimal $k \geqq 0$ such that $\operatorname{deg} R_{k}=\operatorname{deg} R_{k+h}$ for all $h \geqq 0$. $\beta$ is a principal realization of $R_{k}$. Since $M>0$, 
$\bar{k}>0$, and by the minimality of $\bar{k}, \operatorname{deg} R_{\bar{k}-1}>\operatorname{deg} R_{\bar{k}}$. Thus $R_{k}$ is not a generic prolongation of $R_{\bar{k}-1}$. Let $R^{\prime}$ be the generic prolongation $K\left(\beta, \ldots, \beta_{r+\bar{k}-1}, \beta_{r+\bar{k}}^{\prime}\right)$ which specializes to $K\left(\beta, \ldots, \beta_{r+k-1}, \beta_{r+k}\right)$. By the theorem there is a principal realization $\beta^{\prime}$ of $R^{\prime}$ which specializes to $\beta$. $\beta^{\prime}$ is a regular realization of $R$. Furthermore, since the specialization $\beta^{\prime} \vec{K} \beta$ is not generic, $\operatorname{deg} R$-d.t.d. $\left.K\left\langle\beta^{\prime}\right\rangle\right|_{K}<$ $\operatorname{deg} R$-d.t.d. $\left.K\langle\beta\rangle\right|_{K}=M$. By induction there is a principal realization $\alpha$ of $R$ which specializes to $\beta^{\prime}$. Hence $\alpha \underset{K}{\vec{K}} \beta^{\prime} \vec{K} \beta$.

5. Jacobi's bound. Let $A$ be an $n \times n$ matrix $\left|r_{i j}\right|$ where $r_{i j}$ is an integer or $-\infty$. A diagonal sum of $A$ is any sum $r_{1 j_{1}}+r_{2 j_{2}}+\cdots+r_{n j_{n}}$, with $j_{1}, \ldots, j_{n}$ a permutation of $1, \ldots, n$. If $A$ is an $m \times n$ matrix with $M=\min \{m, n\}$, then a diagonal sum of $A$ is a diagonal sum of any $M \times M$ submatrix of $A$. The Jacobi number $J(A)$ of a matrix $A$ is the maximal diagonal sum of $A$.

Two $m \times n$ matrices $A$ and $A^{*}$ are called I-equivalent if $A^{*}$ can be obtained from $A$ by interchanges of rows and interchanges of columns. For $k \leqq \min \{m, n\}$, $A$ will be called $I_{k}$-equivalent to $A^{*}$ if $A^{*}$ can be obtained from $A$ by interchanges within the first $k$ rows and columns. If $A$ and $A^{*}$ are $I_{k}$-equivalent, $J(A)=J\left(A^{*}\right)$.

Proposition 5. Let $A$ be an $m \times n$ matrix $\left|r_{i j}\right|$ with $m>n, r_{i j}=1$ for $i=j$, and $r_{i j}=0$ or 1 for $i \neq j$. Then $A$ is $I_{n}$-equivalent to a matrix of the form:

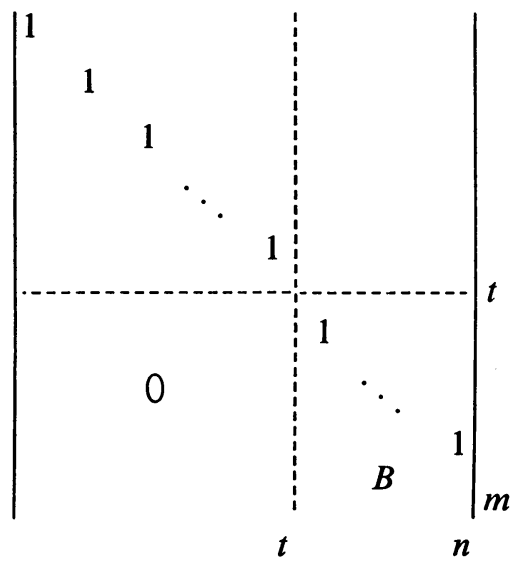

where $0 \leqq t \leqq n$ and for all $j>t$ there is an $i_{j}>j$ such that $r_{i_{j} j}=1$. (Each column of $B$ has a 1 somewhere below the diagonal.)

Proof. We use induction on $\boldsymbol{n}$.

Basis. $n=1$. $A$ is a column $\left|r_{i 1}\right|$ with $r_{11}=1$. Then $A$ has the required form: if $r_{i 1}=0$ for $i>1, t=1$; if $r_{i 1}=1$ for some $i>1, t=0$.

Inductive step. Assume the result holds for any $\bar{m} \times \bar{n}$ matrix $\bar{A}$ with $\bar{m}>\bar{n}$ and $1 \leqq \bar{n}<N$.

Let $A$ be an $m \times N$ matrix, $m>N>1$. Let $C=\left|r_{i j}\right|_{i>N}$ be the submatrix of $A$ consisting of the last $m-N$ rows of $A$. If $C=|0|$, let $t=N$. If $C \neq|0|$, then $r_{p q}=1$ 
for some $p>N$. We may assume that $q=N$ (by interchanges of rows and of columns). Let $D$ be the $m \times(N-1)$ submatrix of $A$ consisting of the first $N-1$ columns of $A$. By induction $D$ is $I_{N-1}$-equivalent to a matrix $D^{*}$ of the required form with $0 \leqq t \leqq N-1$. Since $r_{N N}$ and $r_{p N}$ are unaffected by interchanges within the first $N-1$ rows or $N-1$ columns, $A$ is $I_{N-1}$-equivalent to a matrix $A^{*}$ of the required form with $0 \leqq t \leqq N-1$.

Proposition 6. Let $A$ be an $m \times n$ matrix $\left|r_{i j}\right|$ with $r_{i j}=0$ or 1 . Let $J(A)=J$. Then $A$ is I-equivalent to a matrix of the form:

(3)

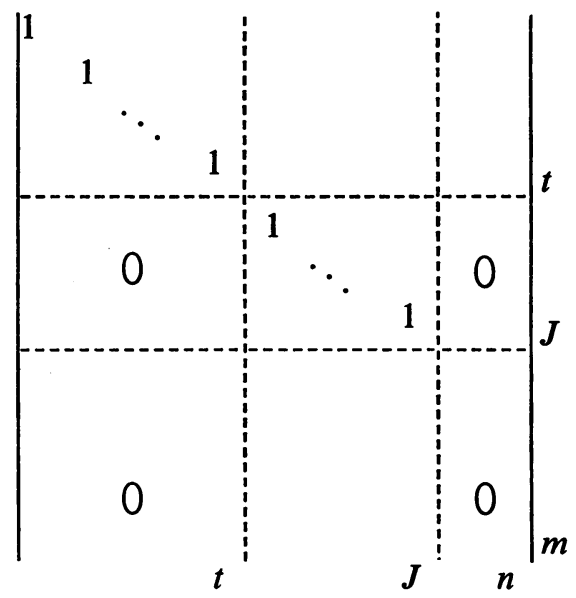

where $0 \leqq t \leqq J$ and for each $j, t<j \leqq J$, there is an $i_{j}>j$ such that $r_{i, j}=1$.

Proof. The case $A=|0|$ is trivial with $0=t=J$; so we assume $A \neq|0|$. Since $J(A)=J$ we may assume that $r_{i i}=1, i=1, \ldots, J$. If $m=J$, let $t=J$. If $m>J$, Proposition 5 may be applied to the submatrix of $A$ consisting of the first $J$ columns of $A$. Hence $A$ is $I_{J}$-equivalent to

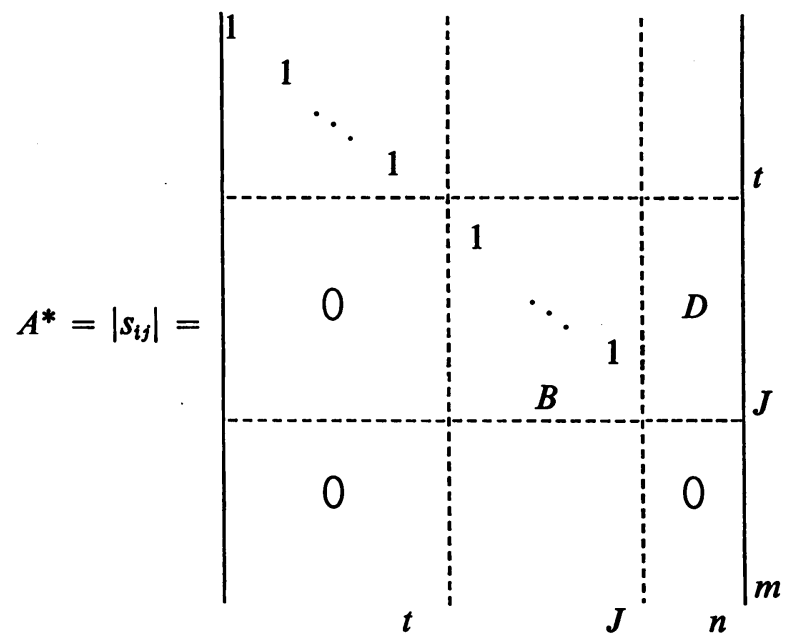


where $0 \leqq t \leqq J$ and each column of $B$ has a 1 in some row below the diagonal. Then it can be shown that $A^{*}$ has form (3). Let $D$ be the submatrix of $A^{*}$ with entries $s_{i j}, t<i \leqq J, J<j \leqq n . D=\varnothing$ if $t=J$ or $J=n$. In either of these cases $A^{*}$ has form (3). If $D \neq \varnothing$, it remains to show that $D=|0|$. If $D \neq|0|$, by using the fact that each column of $B$ has a 1 below the diagonal, one can obtain a diagonal sum greater than $J$, a contradiction. Thus $D=|0|$.

THEOREM 2. Let $K[y]=K\left[y^{(1)}, \ldots, y^{(n)}\right]$ be a polynomial ring over a field $K$. Let $A_{1}, \ldots, A_{m}$ be polynomials of $K[y]$; let $A$ be the $m \times n$ matrix $\left|r_{i j}\right|$ with $r_{i j}=1$ if $y^{(j)}$ appears in $A_{i}$ and $r_{i j}=0$ if not. If the algebraic variety $M\left(A_{1}, \ldots, A_{m}\right)$ is not empty, then every irreducible component has dimension $\geqq n-J(A)$.

Proof. Since interchanging of rows of $A$ corresponds to reordering $A_{1}, \ldots, A_{m}$, and interchanging of columns corresponds to reindexing $y^{(1)}, \ldots, y^{(n)}$, by Proposition 6, we may assume that $A$ is of the form (3), with $J=J(A)$ and $0 \leqq t \leqq J . t+(n-J)$ of the indeterminates do not appear in $A_{t+1}, \ldots, A_{m}$, and thus the irreducible components of $M\left(A_{t+1}, \ldots, A_{m}\right)$ have $\operatorname{dim} \geqq t+n-J$. By the Dimension Theorem [4, p. 36], the components of $M\left(A_{1}, \ldots, A_{t}\right)$ have dimension $\geqq n-t . M\left(A_{1}, \ldots, A_{m}\right)$ $=M\left(A_{1}, \ldots, A_{t}\right) \cap M\left(A_{t+1}, \ldots, A_{m}\right)$. Thus the irreducible components of $M\left(A_{1}, \ldots, A_{m}\right)$ have dimension $\geqq(n-t)+(t+n-J)-n=n-J(A)$.

Let $K[x, y]=K\left[x^{(1)}, \ldots, x^{(m)} ; y^{(1)}, \ldots, y^{(n)}\right]$ be a polynomial ring in $m+n$ indeterminates over a field $K$. If $\Sigma$ is an ideal of $K[x, y]$, let $\Sigma^{\circ}$ denote the ideal $\Sigma \cap K[x]$ in $K[x]$.

Lemma 4. Let $(a, b) \underset{K}{\rightarrow}(\bar{a}, \bar{b})$ where $a=\left(a^{(1)}, \ldots, a^{(m)}\right)$ and $b=\left(b^{(1)}, \ldots, b^{(n)}\right)$. If t.d. $\left.K(\bar{a})\right|_{K}=$ t.d. $\left.K(a)\right|_{K}-s, s \geqq 0$, there exists $c=\left(c^{(1)}, \ldots, c^{(n)}\right)$ such that $(a, b) \vec{K}$ $(\bar{a}, c) \underset{K}{\longrightarrow}(\bar{a}, \bar{b})$ and t.d. $\left.K(\bar{a}, c)\right|_{K} \geqq$ t.d. $\left.K(a, b)\right|_{K}-s$.

Proof. Let $\Pi$ and $\bar{\Pi}$ be the associated ideals of $(a, b)$ and $(\bar{a}, \bar{b})$, respectively, in $K[x, y] \cdot \operatorname{dim} \bar{\Pi}^{\circ}=\operatorname{dim} \Pi^{\circ}-s$. It suffices to obtain a prime ideal $\Lambda$ of $K[x, y]$ such that $\Pi \subseteq \Lambda \subseteq \bar{\Pi}, \Lambda^{\circ}=\bar{\Pi}^{\circ}$, and $\operatorname{dim} \Lambda \geqq \operatorname{dim} \Pi-s$. We use induction on $s$.

Basis. $s=0$. Then $\Pi^{\circ}=\bar{\Pi}^{\circ}$. Let $\Lambda=\Pi$.

Inductive step. Assume that the statement is true for all prime ideals $\Sigma$ and $\bar{\Sigma}$ with $0 \leqq \operatorname{dim} \Sigma^{\circ}-\operatorname{dim} \bar{\Sigma}^{\circ}<S$.

Let $\operatorname{dim} \Pi^{\circ}-\operatorname{dim} \bar{\Pi}^{\circ}=S>0$. Then $\Pi^{\circ} \subset \bar{\Pi}^{\circ}$, and there is an $f(x) \in \bar{\Pi}^{\circ}$ such that $f(x) \notin \Pi^{\circ}$. Hence $\Pi \subset(\Pi, f) \subseteq \bar{\Pi}$, and there is a prime divisor $\Lambda_{1}$ of $(\Pi, f)$ such that $\Pi \subset \Lambda_{1} \subseteq \bar{\Pi}$. By the Dimension Theorem, $\operatorname{dim} \Lambda_{1}=\operatorname{dim} \Pi-1$. Since $f \in \Lambda_{1}^{\circ}, \Pi^{\circ} \subset \Lambda_{1}^{\circ}$ $\subseteq \bar{\Pi}^{\circ}$; thus $\operatorname{dim} \Lambda_{1}^{\circ}-\operatorname{dim} \bar{\Pi}^{\circ}=s_{1} \leqq S-1$. By induction there is a $\Lambda$ such that $\Lambda_{1} \subseteq \Lambda \subseteq \bar{\Pi}, \Lambda^{\circ}=\bar{\Pi}^{\circ}$, and $\operatorname{dim} \Lambda \geqq \operatorname{dim} \Lambda_{1}-s_{1}$. Then $\Pi \subseteq \Lambda \subseteq \bar{\Pi}$, and

$\operatorname{dim} \Lambda \geqq \operatorname{dim} \Lambda_{1}-s_{1} \geqq \operatorname{dim} \Lambda_{1}-(S-1)=\operatorname{dim} \Pi-1-(S-1)=\operatorname{dim} \Pi-S$.

Now let $K$ be a differential field and $K\{y\}=K\left\{y^{(1)}, \ldots, y^{(n)}\right\}$ a differential polynomial ring with a derivation $\partial$. Then $K\left[y, y_{1}\right]$ is a subring of $K\{y\}$, and $\partial$ is a derivation of $K[y]$ into $K\left[y, y_{1}\right]$ with $\partial y=y_{1}$. If $f(y) \in K[y]$, then $\partial(f(y)) \in K\left[y, y_{1}\right]$ and will be denoted by $\partial f\left(y, y_{1}\right)$. 
Let $\Pi$ be a prime ideal of $K\left[y, y_{1}\right]$ with a generic zero $(a, b) . \Pi^{\circ}=\Pi \cap K[y]$ is the ideal associated with $a$ over $K$. Let $G$ be a finite set of generators for $\Pi^{\circ}$. $K(a, b)$ is a kernel if and only if $\partial G \subseteq \Pi[3$, p. 172]. Let the elements of $G$ be indexed $g_{1}(y), \ldots, g_{h}(y)$ so that $\left\{\partial g_{1}\left(a, y_{1}\right), \ldots, \partial g_{r}\left(a, y_{1}\right)\right\}, r \leqq h$, is a maximal linearly independent subset of $\left\{\partial g_{1}\left(a, y_{1}\right), \ldots, \partial g_{h}\left(a, y_{1}\right)\right\}$ over $K(a)$. Then $\partial g_{i}\left(a, y_{1}\right)=$ $\sum_{k=1}^{r} \alpha_{i k} \partial g_{k}\left(a, y_{1}\right), \alpha_{i k} \in K(a), i=1, \ldots, h ;$ and $\partial g_{i}(a, b)=0$ if and only if $\partial g_{k}(a, b)$ $=0, k=1, \ldots, r$. Thus $K(a, b)$ is a kernel if and only if $\partial g_{k}\left(y, y_{1}\right) \in \Pi, k=1, \ldots, r$. When $K(a, b)$ is a kernel,

$$
\left\{\partial g_{i}\left(a, y_{1}\right)=g_{i}^{\partial}(a)+\sum_{j=1}^{n} \frac{\partial g_{i}}{\partial y^{(j)}}(a) \cdot y_{1}^{(j)}, i=1, \ldots, h\right\}
$$

is a consistent system of polynomials in $K(a)\left[y_{1}\right]$ since $b$ is a zero. Since $r$ is the number of linearly independent polynomials, $r$ equals the rank of the matrix

$$
\left|\partial g_{i}(a) / \partial y^{(j)}\right|, \quad i=1, \ldots, h ; j=1, \ldots, n .
$$

But rank $\left|\partial g_{i}(a) / \partial y^{(j)}\right|=n-$ t.d. $\left.K(a)\right|_{K}\left[3\right.$, pp. 177-179]. Therefore, $r=n-$ t.d. $\left.K(a)\right|_{K}$ and $r$ is independent of the choice of generators.

Let $A_{1}, \ldots, A_{m}$ be a system of differential polynomials in $K\{y\}$. Let $r_{i j}$ be the order of $A_{i}$ in $y^{(j)}$, with $r_{i j}=0$ if $y^{(j)}$ does not appear effectively in $A_{i}$. Then the matrix $A=\left|r_{i j}\right|$ of orders may be associated with the system, and the Jacobi number of the system is $J(A)$.

Theorem 3. Let $A_{1}, \ldots, A_{m}$ be first order differential polynomials in $K\{y\}$. Let $A=\left|r_{i j}\right|$ be the matrix of orders $r_{i j}$ of $A_{i}$ in $y^{(j)}$. If $\mathscr{M}$ is an irreducible component of the differential variety $\mathscr{M}\left(A_{1}, \ldots, A_{m}\right)$ with $\operatorname{dim} \mathscr{M}=0$, then ord $\mathscr{M} \leqq J(A)$.

Proof. Let $\bar{\alpha}=\left(\bar{\alpha}^{(1)}, \ldots, \bar{\alpha}^{(n)}\right)$ be a generic zero of $\mathscr{M}$. Then $K\left(\bar{\alpha}, \bar{\alpha}_{1}\right)$ is a kernel $\bar{R}$. Since $\bar{\alpha}$ is a regular realization of $\bar{R}$, it is the specialization of a principal realization $\alpha^{\prime}$ of $\bar{R}$ (Corollary to Theorem 1). But $\alpha^{\prime}$ is a zero of $A_{1}, \ldots, A_{m}$; thus $\bar{\alpha}$ is itself a principal realization. Hence $0=$ d.t.d. $\left.K\langle\bar{\alpha}\rangle\right|_{K}=\operatorname{deg} \bar{R}$, and ord $\mathscr{M}=\operatorname{ord} \bar{R}=$ t.d. $\left.K\left(\bar{\alpha}, \bar{\alpha}_{1}\right)\right|_{K}=$ t.d. $\left.K(\bar{\alpha})\right|_{K}$.

$\left(\bar{\alpha}, \bar{\alpha}_{1}\right)$ is a zero of the ideal $\left(A_{1}, \ldots, A_{m}\right)$ in $K\left[y, y_{1}\right]$. Let $\left(a, a_{1}\right)$ be the generic zero of an irreducible component of the algebraic variety $M\left(A_{1}, \ldots, A_{m}\right)$ such that $\left(a, a_{1}\right) \underset{R}{\vec{K}}\left(\bar{\alpha}, \bar{\alpha}_{1}\right)$. For some $s \geqq 0$,

$$
\text { t.d. }\left.K(\bar{\alpha})\right|_{K}=\text { t.d. }\left.K(a)\right|_{K}-s .
$$

By Lemma 4, there exists $c$ such that $\left(a, a_{1}\right) \underset{K}{\longrightarrow}(\bar{\alpha}, c) \underset{K}{\longrightarrow}\left(\bar{\alpha}, \bar{\alpha}_{1}\right)$, and

$$
\text { t.d. }\left.K(\bar{\alpha}, c)\right|_{K} \geqq \text { t.d. }\left.K\left(a, a_{1}\right)\right|_{K}-s .
$$

Let $\Lambda$ and $\bar{\Pi}$ be the associated prime ideals of $(\bar{\alpha}, c)$ and $\left(\bar{\alpha}, \bar{\alpha}_{1}\right)$, respectively, in $K\left[y, y_{1}\right] . \Lambda^{\circ}=\bar{\Pi}^{\circ}$. Let $\left\{g_{1}(y), \ldots, g_{h}(y)\right\}$ be a set of generators of $\Lambda^{\circ}$ with $\left\{\partial g_{1}\left(\bar{\alpha}, y_{1}\right), \ldots, \partial g_{r}\left(\bar{\alpha}, y_{1}\right)\right\}$ a maximal linearly independent subset of

$$
\left\{\partial g_{i}\left(\bar{\alpha}, y_{1}\right), i=1, \ldots, h\right\}
$$

over $K(\bar{\alpha})$. Since $K\left(\bar{\alpha}, \bar{\alpha}_{1}\right)$ is a kernel, $\partial g_{i}\left(y, y_{1}\right) \in \bar{\Pi}, i=1, \ldots, r$. There exists a prime 
divisor $\Pi$ of the ideal $\left(\Lambda ; \partial g_{1}\left(y, y_{1}\right), \ldots, \partial g_{r}\left(y, y_{1}\right)\right)$ such that $\Pi \subseteq \bar{\Pi} . \operatorname{dim} \Pi$ $\geqq \operatorname{dim} \Lambda-r$. The relation $\Lambda^{\circ} \subseteq \Pi^{\circ} \subseteq \bar{\Pi}^{\circ}=\Lambda^{\circ}$ implies that $\Pi^{\circ}=\bar{\Pi}^{\circ}$. Thus $\Pi$ has a generic zero $\left(\bar{\alpha}, \alpha_{1}\right)$ for some $\alpha_{1}$, and

$$
\text { t.d. }\left.K\left(\bar{\alpha}, \alpha_{1}\right)\right|_{K} \geqq \text { t.d. }\left.K(\bar{\alpha}, c)\right|_{K}-r .
$$

Since $\partial g_{i}\left(y, y_{1}\right) \in \Pi, i=1, \ldots, r, K\left(\bar{\alpha}, \alpha_{1}\right)$ is kernel $R$.

Since $\left(\bar{\alpha}, \alpha_{1}\right) \vec{K}\left(\bar{\alpha}, \bar{\alpha}_{1}\right)$, there is a specialization of the principal realization $\alpha$ of $R$ onto $\bar{\alpha}$ (Theorem 1). But $\alpha$ is a zero of $A_{1}, \ldots, A_{m}$ and $\bar{\alpha}$ is a generic zero of $\mathscr{M}$; therefore, the specialization is generic, and $K\left(\bar{\alpha}, \alpha_{1}\right) \cong_{K} K\left(\bar{\alpha}, \bar{\alpha}_{1}\right)$.

Using this isomorphism, (4), and (5), we obtain

$$
\text { t.d. } \begin{aligned}
\left.K\left(\bar{\alpha}, \bar{\alpha}_{1}\right)\right|_{K} & =\text { t.d. }\left.K\left(\bar{\alpha}, \alpha_{1}\right)\right|_{K} \geqq \text { t.d. }\left.K(\bar{\alpha}, c)\right|_{K}-r \\
& \geqq \text { t.d. }\left.K\left(a, a_{1}\right)\right|_{K}-s-r .
\end{aligned}
$$

Then, since $s=$ t.d. $\left.K(a)\right|_{K}-$ t.d. $\left.K(\bar{\alpha})\right|_{K}$ and t.d. $\left.K(\bar{\alpha})\right|_{K}=$ t.d. $\left.K\left(\bar{\alpha}, \bar{\alpha}_{1}\right)\right|_{K}$, the inequality becomes $0 \geqq$ t.d. $\left.K\left(a, a_{1}\right)\right|_{K(a)}-r$. However, by the remark preceding the theorem, $r=n-$ t.d. $\left.K(\bar{\alpha})\right|_{K}=n-$ ord $\mathscr{M}$. Substitution for $r$ in the above inequality yields

$$
\text { ord } \mathscr{M} \leqq n-\text { t.d. }\left.K\left(a, a_{1}\right)\right|_{K(a)} \text {. }
$$

Let $A_{1}^{*}, \ldots, A_{m}^{*}$ be the polynomials of $K(a)\left[y_{1}\right]$ obtained by substituting $a^{(1)}, \ldots, a^{(n)}$ for $y^{(1)}, \ldots, y^{(n)}$ in $A_{1}, \ldots, A_{m}$. Let $A^{*}$ be the $m \times n$ matrix $\left|r_{i j}^{*}\right|$ with $r_{i j}^{*}=1$ if $y_{1}^{(j)}$ appears in $A_{i}^{*}$, and $r_{i j}^{*}=0$ if not. $J\left(A^{*}\right) \leqq J(A)$ since $r_{i j}^{*}=1$ only if $r_{i j}=1$. By Theorem 2 every component of the algebraic variety $M\left(A_{1}^{*}, \ldots, A_{m}^{*}\right)$ over $K(a)$ has dirnension $\geqq n-J\left(A^{*}\right) \geqq n-J(A)$. $a_{1}$ is a zero of $A_{1}^{*}, \ldots, A_{m}^{*}$; thus there is a generic zero $b$ of a component of $M\left(A_{1}^{*}, \ldots, A_{m}^{*}\right)$ such that $b \underset{K(a)}{\longrightarrow} a_{1}$. But $(a, b)$ is a zero of $A_{1}, \ldots, A_{m}$, and $\left(a, a_{1}\right)$ is a generic zero; therefore the specialization is generic, and t.d. $\left.K\left(a, a_{1}\right)\right|_{K(a)} \geqq n-J(A)$. Using this inequality and (6), one obtains ord $\mathscr{M} \leqq J(A)$.

The following slight generalization can be made in the above theorem. Let $\mathscr{M}$ be an irreducible variety with generic zero $\alpha$. Let $y^{\prime}$ be a subset of the coordinates of $y$. Let $\alpha^{\prime}$ be the coordinates of $\alpha$ corresponding to $y^{\prime}$. Then the order of $\mathscr{M}$ relative to $y^{\prime}$, ord $\left(y^{\prime}\right) \mathscr{M}$, is defined to be t.d. $\left.K\langle\alpha\rangle\right|_{K\left\langle\alpha^{\prime}\right\rangle}$.

Let $B_{1}, \ldots, B_{m}$ be a system of differential polynomials in $K\{y\}$. Let $y^{\prime}$ be a subset of $y$ such that if $y^{(j)} \notin y^{\prime}$, the order $r_{i j}$ of $B_{i}$ in $y^{(j)}$ is 0 or 1 . Let $\left|r_{i j^{*}}\right|$ be the submatrix of the order matrix $\left|r_{i j}\right|$ consisting of those $r_{i j}$ such that $y^{(j)} \notin y^{\prime}$. If $\mathscr{M}$ is a component of $\mathscr{M}\left(B_{1}, \ldots, B_{m}\right)$ for which $y^{\prime}$ contains a complete set of parameters, then

$$
\text { ord }\left(y^{\prime}\right) \mathscr{M} \leqq J\left(r_{i j^{*}}\right) \text {. }
$$

This theorem can be reduced to Theorem 3 by a method similar to that of Theorem IX, Chapter 8, of [2].

Note 3. Any finite system $\mathscr{S}$ of differential polynomials may be reduced to an equivalent first order system $\mathscr{T}$. However the Jacobi number of $\mathscr{T}$ may be greater than that of $\mathscr{S}$. Thus the extension of Theorem 3 to arbitrary systems is not 
immediate. This situation suggests that the strong Jacobi number might be a more natural bound. Let $\mathscr{S}$ consist of $A_{1}, \ldots, A_{m}$ with an order matrix $\left|r_{i j}\right|$. We may define the strong order $s_{i j}$ of $A_{i}$ in $y^{(j)}$ to be $r_{i j}$ if $y^{(j)}$ is effectively present in $A_{i}$, and to be $-\infty$, if not. Then $J^{\prime}=J\left(s_{i j}\right)$ is the strong Jacobi number of the system $\mathscr{S}$. $J^{\prime} \leqq J\left(r_{i j}\right)$. It can be shown that the strong Jacobi number of $\mathscr{T}$ is equal to that of $\mathscr{S}$.

6. Examples. Let $\boldsymbol{A}$ be an $\boldsymbol{n} \times \boldsymbol{n}$ matrix of nonnegative integers. It will be shown that there exists a system of $n$ differential polynomials in $n$ indeterminates with a matrix of order equal to $A$ such that some zero-dimensional component of the variety of the system has order equal to $J(A)$. Thus, if the Jacobi number is a bound (as it is for first order systems), then it is a bound achieved by some system. A more general result is easily obtained by taking $A$ to be a matrix with entries that are nonnegative integers or $-\infty$, and such that $J(A) \geqq 0$. A system can then be found with order equal to the strong Jacobi number.

Let $K$ be a differential field with $L$ the subfield of constants under the derivation $\partial$. Let $L \neq K$. Let $L[\partial]$ be the ring of linear differential operators. Let $\Delta$ denote an $n \times n$ matrix $\left|f_{i j}(\partial)\right|$ of such operators, $y$ the vector

$$
\left|\begin{array}{l}
y^{(1)} \\
\vdots \\
y^{(n)}
\end{array}\right|,
$$

and $\Delta y=0$ the system of linear differential equations

$$
\left\{A_{i}=f_{i 1}(\partial) y^{(1)}+\cdots+f_{i n}(\partial) y^{(n)}=0, i=1, \ldots, n\right\} .
$$

Let $s_{i j}$ denote the strong order of $A_{i}$ in $y^{(j)}$. Then $s_{i j}=\operatorname{deg} f_{i j}(\partial)$, the degree of $f_{i j}(\partial)$ as a polynomial in $\partial \cdot \operatorname{deg}(\operatorname{det} \Delta) \leqq J\left(\operatorname{deg} f_{i j}(\partial)\right)=J\left(s_{i j}\right)$.

Let $A$ be an $n \times n$ matrix with entries that are nonnegative integers or $-\infty$, and let $J(A) \geqq 0$. Let $A=\left|s_{i j}\right|$. If $s_{i j}=s \neq-\infty$, let $F_{i j}(x, \partial)=x_{i j 0}+x_{i j 1} \partial+\cdots+x_{i j s} \partial^{s}$, where the $x_{i j k}$ are indeterminates. If $s_{i j}=-\infty$, let $F_{i j}(x, \partial)=0$. Then $\operatorname{det}\left|F_{i j}(x, \partial)\right|$ is a polynomial $p_{0}(x)+\cdots+p_{m}(x) \partial^{m}$, with $m=J\left(s_{i j}\right) \geqq 0$ and with each $p_{h}(x)$ a polynomial over the integers in the indeterminates $x_{i j k}$. There exists a point $\left(a_{i j k}\right)$ of integers such that $p_{m}(a) \neq 0$. Let $f_{i j}(\partial)=F_{i j}(a, \partial) \in L[\partial]$, and let $\Delta=\left|f_{i j}(\partial)\right|$. $\left|\operatorname{deg} f_{i j}(\partial)\right|=A$ and $\operatorname{deg}(\operatorname{det} \Delta)=m=J(A)$. The system $\Delta y$ has strong Jacobi number $J^{\prime}=J(A)$. It will be shown that this system has a component of dimension zero and order $J(A)$.

There exists a matrix $\Lambda$ of linear differential operators such that det $\Lambda \in L$ and $\Lambda \Delta$ has a triangular form:

$$
\left|\begin{array}{cccc}
h_{11}(\partial) & h_{12}(\partial) & \ldots & \\
0 & h_{22}(\partial) & h_{23}(\partial) & \ldots \\
0 & 0 & h_{33}(\partial) & \ldots \\
\vdots & & & \\
0 & \ldots & 0 & h_{n n}(\partial)
\end{array}\right|
$$


with $h_{i i}(\partial) \neq 0$ for all $i$. The method of obtaining $\Lambda$ may be indicated as follows. Since det $\Delta \neq 0, f_{i 1}(\partial) \neq 0$ for some $i$. We may assume that $f_{11}(\partial) \neq 0$ and $f_{21}(\partial) \neq 0$. Let $g(\partial)=$ g.c.d. $\left(f_{11}, f_{21}\right)$. Then $f_{11}(\partial)=g(\partial) \cdot d(\partial)$ and $f_{21}(\partial)=g(\partial) \cdot e(\partial)$, and $e f_{11}-d f_{21}=0$. Since $d$ and $e$ are relatively prime, there exist $p(\partial)$ and $q(\partial)$ in $L[\partial]$ such that $d p+e q=1$. Let

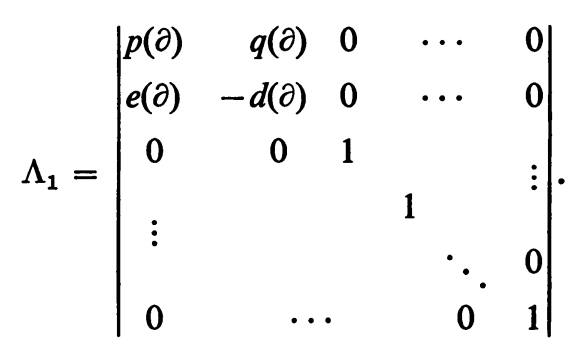

Then det $\Lambda_{1}=-1$ and

$$
\Lambda_{1} \Delta=\left|\begin{array}{ccccc}
g_{11}(\partial) & g_{12}(\partial) & \ldots & & \\
0 & g_{22}(\partial) & g_{23}(\partial) & \ldots & \\
f_{31}(\partial) & f_{32}(\partial) & f_{33}(\partial) & \cdots & f_{3 n}(\partial) \\
\vdots & & & \ddots & \vdots \\
f_{n 1}(\partial) & \ldots & & & f_{n n}(\partial)
\end{array}\right|
$$

Since det $\left(\Lambda_{1} \Delta\right)=-\operatorname{det} \Delta \neq 0$, some entry in the first column is nonzero. This procedure may be repeated to obtain matrices $\Lambda_{i}$. Eventually the product $\Lambda$ of such $\Lambda_{i}$ will satisfy the requirements. Since det $\Lambda \in L, \Lambda$ has an inverse $\Lambda^{-1}$ in $L[\partial]$. Thus the systems $\Delta y$ and $\Lambda \Delta y$ are equivalent. $\operatorname{det} \Lambda \Delta=c \operatorname{det} \Delta, c \in L$. Hence $\operatorname{deg}(\operatorname{det} \Lambda \Delta)=\operatorname{deg}(\operatorname{det} \Delta)=J(A)$. A zero $\left(\alpha^{(1)}, \ldots, \alpha^{(n)}\right)$ of $\Lambda \Delta y$ (and in fact the generic zero of a component) may be obtained by successively solving one equation in one indeterminate, beginning with the last.

$$
\text { t.d. }\left.K\left\langle\alpha^{(i)}, \ldots, \alpha^{(n)}\right\rangle\right|_{K\left\langle\alpha^{(i+1)}, \ldots, \alpha^{(n)}\right\rangle}=\operatorname{deg} h_{i i}(\partial)
$$

Thus d.t.d. $\left.K\langle\alpha\rangle\right|_{K}=0$, and t.d. $\left.K\langle\alpha\rangle\right|_{K}=\sum_{i=1}^{n} \operatorname{deg} h_{i i}(\partial)=\operatorname{deg}(\operatorname{det} \Lambda \Delta)=J(A)$.

\section{REFERENCES}

1. C. Chevalley, Introduction to the theory of algebraic functions of one variable, Math. Surveys, no. 6, Amer. Math. Soc., Providence, R. I., 1951. MR 13, 64.

2. R. M. Cohn, Difference algebra, Interscience, New York, 1965. MR 34 \#5812.

3. N. Jacobson, Lectures in abstract algebra. Vol. III: Theory of fields and Galois theory, Van Nostrand, Princeton, N. J., 1964. MR 30 \#3087.

4. S. Lang, Introduction to algebraic geometry, Interscience, New York, 1958. MR 20 \#7021. 
5. J. F. Ritt, Jacobi's problem on the order of a system of differential equations, Ann. of Math. 36 (1935), 303-312.

6. —, Differential algebra, Amer. Math. Soc. Colloq. Publ., vol. 33, Amer. Math. Soc., Providence, R. I., 1950. MR 12, 7.

7. O. Zariski and P. Samuel, Commutative algebra. Vol. I, University Series in Higher Math., Van Nostrand, Princeton, N. J., 1958. MR 19, 833.

8. — Commutative algebra. Vol. II, University Series in Higher Math., Van Nostrand, Princeton, N. J., 1960. MR 22 \#11006.

RUTGERS UNIVERSITY,

New BRUNSWiCK, New JeRSEy 08903 